\title{
Sirtuin 6 in preosteoclasts suppresses age- and estrogen deficiency-related bone loss by stabilizing estrogen receptor a
}

\author{
Young Jae Moon ${ }^{1}$ Zhongkai Zhang ${ }^{2}$ - In Hyuk Bang ${ }^{1}$. Oh Kwang Kwon ${ }^{3}$. Sun-Jung Yoon ${ }^{2}$ Jung Ryul Kim² \\ Sangkyu Lee ${ }^{3} \cdot$ Eun Ju Bae ${ }^{4}$ Byung-Hyun Park ${ }^{1}$
}

Received: 20 December 2018 / Revised: 1 February 2019 / Accepted: 4 February 2019 / Published online: 20 February 2019

(c) ADMC Associazione Differenziamento e Morte Cellulare 2019

\begin{abstract}
Emerging evidence indicates that estrogen receptor (ER) $\alpha$ is an important modulator of bone homeostasis, which occurs partly by promoting osteoclast apoptosis. Sirtuin 6 (Sirt6) is an anti-aging molecule, and its deficiency in mice results in skeletal malformations associated with progeroid features. However, the effects of Sirt6 on ER $\alpha$ function in osteoclasts, and thus on aging- or estrogen deficiency-induced bone loss, have not been studied. Here, we show that myeloid-specific deletion of Sirt6 led to decreased ER $\alpha$ protein level and apoptotic cell death in preosteoclasts. Consequently, myeloid Sirt6 KO mice showed aggravated cancellous bone loss both with aging and following an ovariectomy compared to wild-type littermates. In contrast, Sirt6 transgenic mice were protected from ovariectomy-induced bone loss. Mechanistically, Sirt6 deacetylated ER $\alpha$ protein to prevent its proteasomal degradation, in which lysine 171 and lysine 299 were critical residues. Sirt6-mediated ER $\alpha$ stabilization promoted transcription of Fas ligand in preosteoclasts, resulting in apoptosis of osteoclasts. Finally, the level of Sirt6 in human preosteoclasts was correlated positively with bone density and ER $\alpha$ but negatively with age. In conclusion, our results suggest that deacetylation and upregulation of ER $\alpha$ by Sirt6 in preosteoclasts prevent bone loss by inhibiting osteoclast-mediated bone resorption. Activation of Sirt6 in preosteoclasts may provide a new therapeutic approach to attenuate osteoporosis in older or postmenopausal patients.
\end{abstract}

Edited by D. Vaux

Supplementary information The online version of this article (https:// doi.org/10.1038/s41418-019-0306-9) contains supplementary material, which is available to authorized users.

Eun Ju Bae

ejbae@woosuk.ac.kr

$\triangle$ Byung-Hyun Park

bhpark@jbnu.ac.kr

1 Department of Biochemistry and Molecular Biology, Chonbuk National University Medical School, Jeonju, Jeonbuk 54896, Republic of Korea

2 Department of Orthopaedic Surgery, Chonbuk National University Medical School, Jeonju, Jeonbuk 54896, Republic of Korea

3 BK21 Plus KNU Multi-Omics based Creative Drug Research Team, College of Pharmacy, Kyungpook National University, Daegu 41566, Republic of Korea

4 College of Pharmacy, Woosuk University, Wanju, Jeonbuk 55338, Republic of Korea

\section{Introduction}

Osteoporosis is a disease characterized by loss of bone mass and degeneration of bone microstructure, leading to increased risk of fracture. The acute loss of estrogen during menopause or after ovariectomy (OVX) accelerates the rate of bone remodeling, with bone resorption by osteoclasts exceeding bone formation by osteoblasts, resulting in a net bone loss [1]. While this estrogen-centric view provides a rationale for estrogen replacement therapy, epidemiological studies in humans and mechanistic studies in animals indicate that aging plays a pivotal role in bone loss irrespective of estrogen deficiency. To support this notion, a follow-up cohort study of the National Health and Nutrition Examination Survey has reported that bone loss begins as early as the third decade, long before the decline in sex steroid production in both women and men [2]. Similarly, osteoporotic changes begin at 4-8 months of age and last until 31 months of age in C57BL/6 mice, which do not experience menopause with aging [3]. Nevertheless, hyperactivation of 
osteoclasts is the hallmark of both postmenopausal and senile osteoporosis.

Osteoclasts differentiate from hematopoietic cells in response to stimulation by macrophage colony-stimulating factor (M-CSF) and receptor activator of NF- $\mathrm{BB}$ ligand (RANKL). One of the regulatory mechanisms used by bone cells to defend against osteoclastic bone resorption involves apoptosis, which determines the lifespan and number of osteoclasts. Estrogen protects against bone loss by regulating osteoclast survival. Nakamura et al. [4] demonstrated that estrogen increases Fas ligand (FasL) expression in osteoclasts of cancellous bones to induce auto-apoptosis. In a separate study, Krum et al. [5] showed that estrogen stimulates FasL expression in osteoblasts to induce osteoclastic apoptosis through a paracrine mechanism. These two studies clearly demonstrate that appropriate apoptosis of osteoclasts is crucial for maintenance of bone density. The effects of estrogen on osteoclasts are mediated primarily via their own receptors, estrogen receptor (ER) $\alpha$ and $\beta$. Studies using osteoclastspecific ER $\alpha$ knockout (KO) mice show that binding of estrogen to ER $\alpha$ enhances apoptotic cell death of osteoclasts and increases cancellous bone density $[4,6]$.

In addition to estrogen binding, the transcriptional activity of ER $\alpha$ is also regulated by posttranslational modifications such as acetylation, phosphorylation, sumoylation, and palmitoylation [7-10]. Among these modifications, acetylation has emerged as a central step in regulatory modification of ER $\alpha$. Acetylation occurs on multiple lysine residues (K266, K268, K302, and K303) in the hinge region of ER $\alpha$ [11, 12], and the functional consequences of acetylation are diverse. Acetylation of ER $\alpha$ at K266 and K268 enhances the DNA binding and transcriptional activities of ER $\alpha$ in a ligand-dependent manner [11], whereas acetylation at K302 and K303 suppresses the ligand sensitivity of ER $\alpha$ [12]. Previous reports have shown that acetylation of ER $\alpha$ positively regulates its transcriptional activity by either potentiating its response to ligand [11] or by enhancing its stability [13].

Protein acetylation is reciprocally controlled by the actions of histone acetyltransferases and deacetylases. Sirtuins (Sirt1 to Sirt7) are highly conserved NAD ${ }^{+}$-dependent deacetylases that regulate lifespan in lower organisms and age-related diseases in mammals [14]. Genetic deletion of Sirtl in osteoclast precursor cells decreases bone mass $[15,16]$, and pharmacological activation of Sirt1 prevents the bone loss caused by OVX [17] or aging [18]. However, the effects of Sirt6, a nuclear form of sirtuin, on estrogen deficiency- or age-related osteoporosis have not been reported. In this study, we investigated the role of Sirt6 in preosteoclasts on regulation of bone mass in osteoporosis animal models and identified its mechanism of action.

\section{Materials and methods}

\section{Animal experiments}

Sirt6 transgenic mice (C57BL/6-Tg(RP23-352G18)1Coppa/J), Sirto ${ }^{f l f l}$ mice $\left(\mathrm{B} 6 ; 129-\right.$ Sirt $^{\mathrm{tm} 1 \mathrm{Ygu} / \mathrm{J})}$, and LysM-Cre mice (B6.129P2-lyz $\left.2^{\mathrm{tm1}(\mathrm{cre}) / \mathrm{fo}} / \mathrm{J}\right)$ were obtained from Jackson Laboratory (Bar Harbor, ME, USA). The mS6KO mice $\left(\operatorname{Sirto}^{f l f l}:\right.$ LysM $)$ were generated as described previously [26]. Bilateral OVX or sham operation was performed on 12-week-old female mice, and the mice were sacrificed after 6 weeks. Bone parameters were analyzed using microcomputed tomography $(\mu \mathrm{CT})$. The study protocol was approved by the Institutional Animal Care and Use Committee of Chonbuk National University (Permit No: CBNU 2015-8).

\section{Patients}

Patients who underwent hip arthroplasty in Chonbuk National University Hospital (Jeonju, Korea) between August 2017 and August 2018 were evaluated in this study. The inclusion criteria were as follows: men or women over 18 years old; patients who evaluated BMD either before or after the operation; patients with adequate bone marrow cells extraction from femoral neck cutting area; and when extracted bone marrow cells were not contaminated. Those with neuromuscular disorder, rheumatoid arthritis, endocrinological disease, revision arthroplasty, disease that affected bone metabolism (or those taking drugs that affected bone metabolism), and past hormone replacement therapy were excluded from the study. According to these criteria, 24 patients met criteria. The severe osteoporosis group was defined as BMD T-score below -2.5 together with femoral neck fractures due to minor trauma. The control group comprised osteoarthritis patients. The demographic and clinical characteristics of the control and severe osteoporosis patients included in this study were shown in Table S1. Either before or after the operation, BMD was determined at femoral neck using dual-energy X-ray absorptiometry (GE Lunar, Madison, WI, USA) for all patients, and serum CTX was measured in 16 patients. The control group comprised osteoarthritis patients, and the severe osteoporosis group was defined as BMD T-score below -2.5 together with one or more fractures. The study protocol was approved by the Institutional Review Board of Chonbuk National University Hospital (IRB No. 2017-09-021).

\section{Histology}

Femurs were fixed in $4 \%$ paraformaldehyde and decalcified in $15 \%$ ethylenediaminetetraacetic acid solution for 8 weeks. 
Decalcified femoral bones were then dehydrated, embedded in paraffin, and sectioned $(5 \mu \mathrm{m})$. After deparaffinization, the sections were stained with hematoxylin and eosin (H\&E) and tartrate-resistant acid phosphatase (TRAP, Sigma-Aldrich, St. Louis, MO, USA).

\section{In vitro osteoclastogenesis}

Osteoclastogenesis was performed with $\mathrm{CD}_{11 \mathrm{~b}}{ }^{+}$bone marrow cells (BMCs) from femora of 12-week-old mice. The cells were seeded in 6-well plates and cultured with $30 \mathrm{ng} / \mathrm{ml}$ M-CSF (PeproTech, London, England) for 2 days. Osteoclast formation was induced through medium replacement with growth medium containing M-CSF (10 ng/ml) and RANKL (30 ng/ml, PeproTech). TRAP ${ }^{+}$multinuclear cells were detected using a TRAP staining kit (SigmaAldrich) at 3 or 4 days after M-CSF and RANKL treatment. Apoptotic cells were detected using the APOPercentage apoptosis assay kit (Biocolor, Belfast, Northern Ireland).

For induction of osteoclasts from human BMCs, CD11 ${ }^{+}$ human BMCs were seeded at $2 \times 10^{6}$ cells/well in 12-well plates and cultured with $25 \mathrm{ng} / \mathrm{ml} \mathrm{M-CSF}$ and $50 \mathrm{ng} / \mathrm{ml}$ RANKL for 7 days. Half of the culture medium, supplemented with cytokines (M-CSF and RANKL), was replaced on days 3 and 6.

\section{Cytokine detection}

The serum levels of RANKL (Abcam, Cambridge, MA, USA) and osteoprotegerin (OPG, R\&D Systems, MN, USA) were detected using ELISA kits according to the manufacturer's instructions.

\section{Cell culture and transient transfection}

The human embryonic kidney cell line HEK293T was obtained from the American Type Culture Collection (Manassas, VA, USA). Exogenous proteins were expressed by transfecting HEK293T cells with $1 \mu \mathrm{g}$ of plasmid DNA containing Flag, Flag-Sirt6, Flag-Sirt6-H133Y (mutant Sirt6), or HA-Esrl using Lipofectamine 3000 (Invitrogen, Carlsbad, CA, USA). To evaluate promoter activity, $1 \mu \mathrm{g}$ each of FasL, ERE, and AP-1 promoter luciferase (Promega, Madison, WI, USA) were used.

\section{Antibodies}

Antibodies against the following proteins were purchased from the manufacturer indicated: ER $\alpha$, Ub, FasL (Santa Cruz Biotechnology, Dallas, TX, USA), Sirt6, cleaved caspase 8, cleaved caspase 3, Bax, Ac-Lys (Cell Signaling Technology, Beverly, MA, USA), NFATc1 (Abcam), HA (Roche Diagnostics, Mannheim, German), HSP90 (Enzo
Life Sciences, Plymouth Meeting, PA, USA), $\alpha$-tubulin, and GAPDH (Bioworld Technology, St. Louis Park, MN, USA).

\section{Liquid chromatography (LC)-mass spectrometry (MS/MS) analysis}

Immunoprecipitated proteins were visualized by staining SDS gels with colloidal Coomassie blue. After the proteins were excised from the gel bands, they were subjected to ingel trypsin digestion. The tryptic peptides were analyzed for acetylation using a high-resolution mass spectrometer (LTQ-Orbitrap Velos, Thermo Fisher Scientific, Waltham, MA, USA) coupled with nano-flow liquid chromatograph (nano-LC) as described previously [27].

\section{Site-directed mutagenesis}

Acetylation mutants of $\mathrm{ER} \alpha$ were generated using a sitedirected mutagenesis kit (GeneAll, Seoul, Korea) by converting each lysine residue (K171 and K299) to arginine (codon change from AAG to AGG). Mutations were confirmed with DNA sequencing performed at Cosmogenetech (Seoul, Korea).

\section{Statistical analysis}

The data are expressed as the mean \pm standard error of the mean (SEM). Statistical comparisons were performed using one-way analysis of variance followed by Fisher's post hoc analysis. The significance of differences between two groups was determined using Student's unpaired $t$-test. Linear regressions were performed with GraphPad Prism, version 5.02 (San Diego, CA, USA). A $p$-value $<0.05$ was considered significant.

\section{Additional methods}

Detailed methods are provided in the Supplementary Materials and Methods.

\section{Results}

\section{Sirt6 expression and apoptosis are attenuated in old preosteoclasts}

To determine whether sirtuins play a role in aging-related bone loss, we isolated $\mathrm{CD}_{11} \mathrm{~b}^{+}$bone marrow cells (BMCs) from young (3 months) and old (20 months) mice. CD11b ${ }^{+}$ BMCs were cultured in the presence of M-CSF and RANKL. Upon osteoclastogenesis induction, Sirt6 protein expression was significantly decreased in $\mathrm{CD}_{11 b^{+}} \mathrm{BMCs}$ 
from old mice compared to those from young mice; however, the expression of other sirtuins was unaffected (Fig. 1a), implying the involvement of Sirt6 as an inhibitory regulator of aging-induced bone loss. Since deletion of Sirt6 increases resistance to apoptotic cell death [19], and ER $\alpha$ mediated FasL expression is closely related to apoptosis in osteoclasts $[4,5]$, we questioned if Sirt6 reduction in old $\mathrm{CD}_{1} 1 \mathrm{~b}^{+}$BMCs could affect ER $\alpha$ and FasL expression in osteoclasts. In contrast to the results from young CD11 ${ }^{+}$ BMCs in which M-CSF and RANKL treatment markedly increased the protein levels of ER $\alpha$ and FasL, little change was observed in old CD11 $b^{+}$BMCs (Fig. 1b). mRNA level of ER $\alpha$ (Esrl) was not different between young and old $\mathrm{CD}_{11 \mathrm{~b}^{+}}$BMCs (Fig. 1c), suggesting that the change by aging in $E R \alpha$ protein in preosteoclasts occurred at the posttranscriptional level. Consistent with the reduced FasL expression, less apoptosis and more osteoclastogenesis were observed in old CD11 $\mathrm{b}^{+}$BMCs compared to young mice (Fig. 1d, e).

To better understand the involvement of the Sirt6-ER $\alpha$ FasL pathway in osteoclastic bone resorption, CD11b BMCs isolated from 3-month-old mice were treated with
M-CSF and RANKL for 4 days. Time course expression analyses showed that the highest expression of Sirt6, ER $\alpha$, and FasL was observed at day 3, and that this increase occurred exclusively in the nuclear fraction of cells (Figs. $\mathrm{S} 1 \mathrm{a}$ and S1b). The protein and mRNA levels of mature osteoclast markers, such as NFATc1 and Calcr, were delayed in comparison to the peak expression of Sirt6 (Fig. S1a and S1c), suggesting that osteoclastogenic stimuli activate the Sirt6-ER $\alpha$-FasL pathway at the preosteoclast stage.

\section{Myeloid Sirt6 deficiency augments aging- and ovariectomy-induced cancellous bone loss in mice}

To examine the effects of selective deletion of myeloid Sirt6 on osteoclastogenesis and other aspects of bone metabolism in vivo, we generated $\mathrm{mS} 6 \mathrm{KO}$ mice and compared the bone mass of old WT and KO mice. The mS6KO mice and their wild-type littermates had phenotypically normal skeletons at birth and similar bone mass at 3 months of age (data not shown). However, at 20 months of age, the mS6KO mice had increased cancellous bone loss as revealed using $\mu \mathrm{CT}$

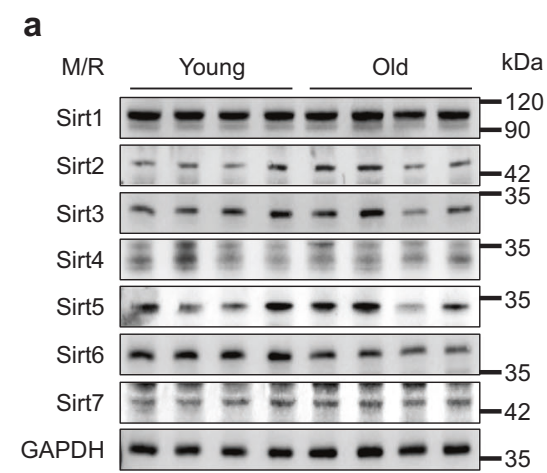

d
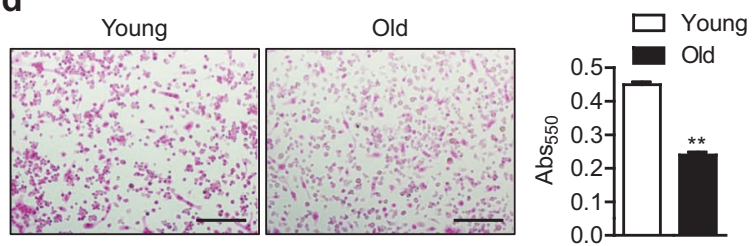

Fig. 1 Reduction of Sirt6 expression and attenuation of apoptosis in $\mathrm{CD} 11 \mathrm{~b}^{+}$BMCs derived from old mice. a CD $11 \mathrm{~b}^{+} \mathrm{BMCs}$ from young (3 months) and old (20 months) mice were treated with M-CSF $(10 \mathrm{ng} / \mathrm{ml})$ and RANKL $(30 \mathrm{ng} / \mathrm{ml})$ for 3 days, and sirtuin protein level was determined. The band intensities were quantified using densitometry $(n=4)$. M/R, M-CSF plus RANKL. b, c CD11b BMCs from young and old mice were treated with M-CSF and RANKL for 3 days, and proteins and mRNA levels were analyzed using western blotting and real-time RT-PCR, respectively b

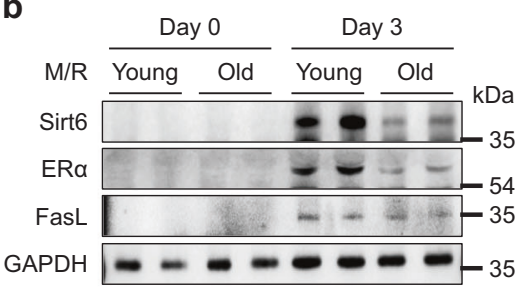

C
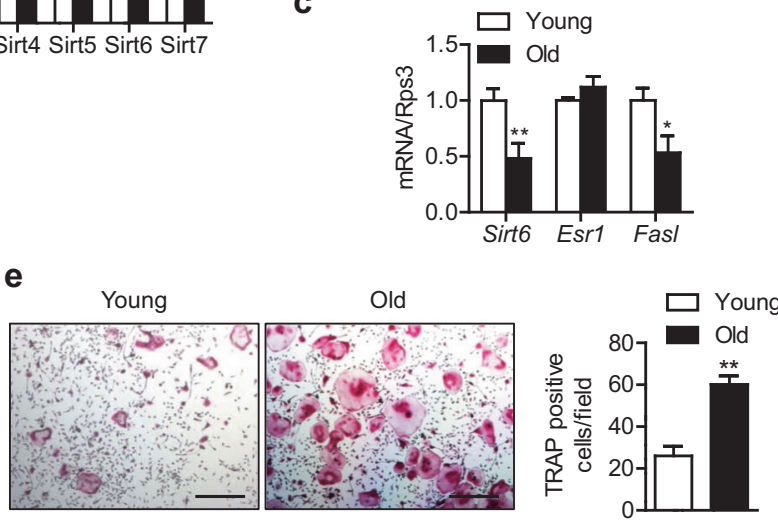

( $n=6-10)$. d Apoptosis in osteoclasts was assessed using the APOPercentage apoptosis assay kit. Scale bars $=100 \mu \mathrm{m}$. Apoptotic cells appear bright pink. Absorbance of the accumulated pink dye was measured with a spectrophotometer at $550 \mathrm{~nm}(n=6)$. e Representative TRAP staining after osteoclastogenesis induction. Scale bars $=200 \mu \mathrm{m}$. TRAP-positive multinucleate cells with three or more nuclei were counted as osteoclasts and were scored per field $(n=5)$. The values are mean \pm SEM. $* p<0.05$ and ${ }^{* *} p<0.01$ versus young 
Fig. 2 Aggravation of agerelated cancellous bone loss by Sirt6 deficiency. a, b Uterine weight $(n=4)$ and RANKL per OPG ratio in serum $(n=7)$ were compared in 20-month-old mS6KO and WT mice. c, $\mathbf{d}$ Representative $\mu \mathrm{CT}$ reconstruction and bone morphometric parameters of the distal femur of 20-month-old $\mathrm{mS6KO}$ and WT mice $(n=5)$. $\mathrm{BV}$ bone volume, TV tissue volume, $\mathrm{Tb}$.Th trabecular thickness, Tb.N trabecular number, Tb.Sp trabecular separation. e Representative $\mathrm{H} \& \mathrm{E}$ staining (scale bars $=$ $250 \mu \mathrm{m})$ and TRAP staining (scale bars $=50 \mu \mathrm{m}$ ) of distal femurs. Osteoclast numbers per bone surface (Oc.N/BS) and the ratio of osteoclast surface to bone surface (Oc.S/BS) were determined $(n=8-11)$, and the values are mean \pm SEM. $* * p<$ 0.01 versus WT
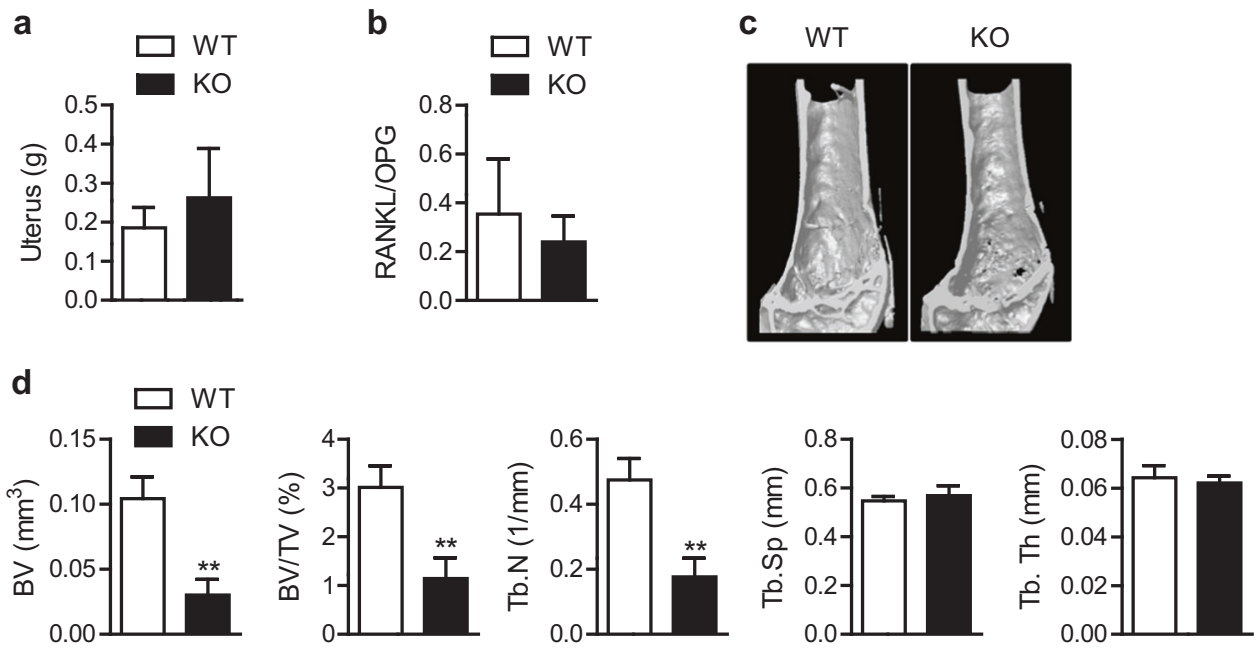

e
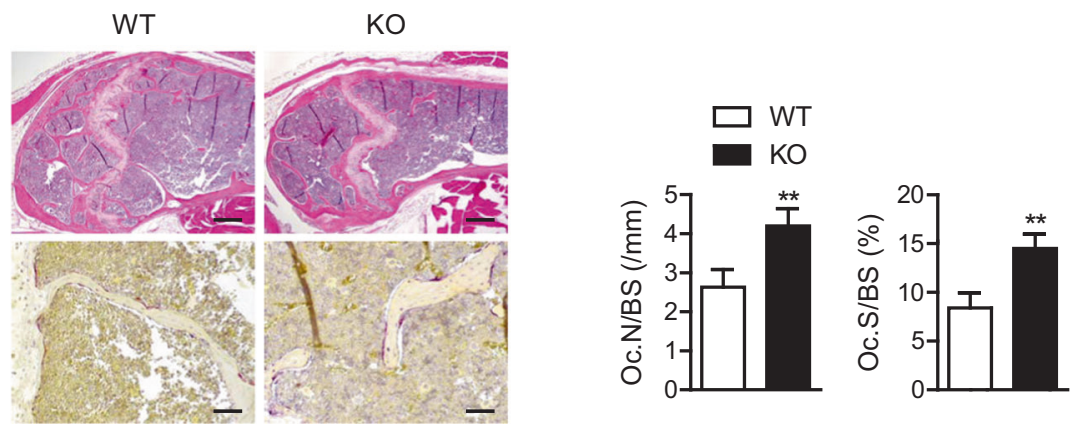

imaging of the distal femur and lumbar spine (Fig. 2c and Fig. S2a), although uterine weight and RANKL/OPG ratio, which are indexes of estrogen level and osteoclastogenic stimuli, respectively, were not different between the genotypes (Fig. 2a, b). Consistently, BV, BV/TV, and Tb.N were significantly lower in the mS6KO mice compared to the WT group (Fig. 2d and Fig. S2b). H\&E analysis confirmed the $\mu \mathrm{CT}$ results (Fig. 2e). To further examine whether the decreased bone mass was due to an increase in the number of osteoclasts, we performed TRAP staining on the cancellous bone of the distal femur and found that myeloid Sirt6 deficiency increased the number of mature osteoclasts by twofold compared with the WT mice (Fig. 2e). However, Sirt6 deficiency caused little change in cortical bone (Fig. S3).

Because high levels of osteoclast-mediated resorption are due not only to aging, but also to sex hormone deficiency $[1,20]$, we next carried out bilateral OVX in WT and mS6KO female mice. Successful OVX was confirmed by decrease in uterine weight and increase in RANKL/OPG ratio, which showed no differences between the genotypes (Fig. 3a, b). H\&E staining and $\mu \mathrm{CT}$ analysis of the distal femur revealed that cancellous bone loss in mS6KO mice was much greater than in WT mice (Fig. 3c, d, and Fig. S4). Consistently, the number of osteoclasts was highly increased in mS6KO mice compared with WT mice (Fig. 3e). Sirt6 deficiency caused little change in cortical bone after OVX (Fig. S5), confirming that myeloid Sirt6 deletion selectively affects cancellous bone in both agingand OVX-induced bone loss.

\section{ERa/FasL-mediated apoptotic cell death is reduced in $\mathrm{mS6KO}$ mice and aged mice but is increased by re-expression of Sirt6}

It is possible that downregulation of ER $\alpha$ and FasL in preosteoclasts by Sirt6 deletion inhibits apoptosis. To address this issue, we compared FasL-initiated apoptosis in preosteoclasts from $\mathrm{WT}$ and $\mathrm{mS6KO}$ mice. Induction of $\mathrm{ER} \alpha$ and FasL in preosteoclasts upon stimulation with M-CSF and RANKL was lower in KO mice compared to WT mice. Correspondingly, the expression of downstream molecules involved in apoptosis was also attenuated in $\mathrm{KO}$ mice (Fig. 4a). However, these effects were not related to the transcriptional changes in the estrogen receptors Esrl and Esr2 or the FasL receptor Fas (Fig. 4b), implying that posttranscriptional regulation of ER $\alpha$ by Sirt6 could be a cause of apoptotic alteration. The staining results for apoptotic and TRAP-positive cells further supported these results (Fig. 4c, d). 
a

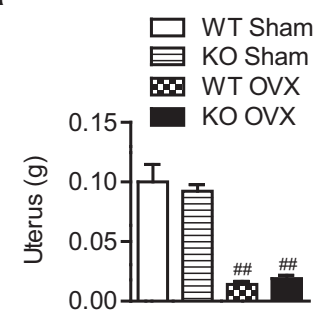

d

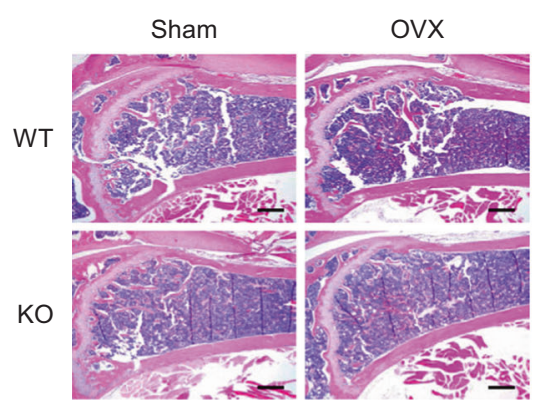

b

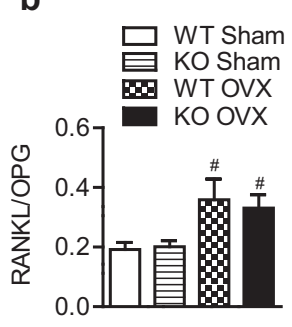

C

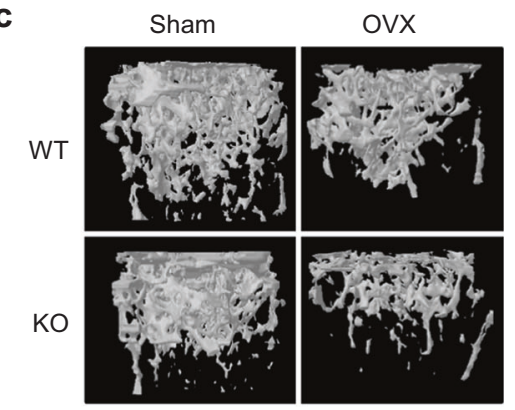

e

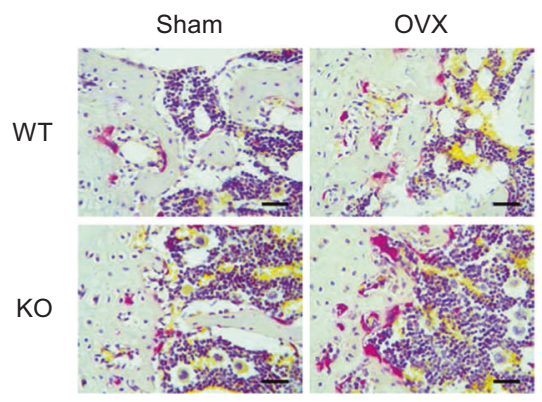

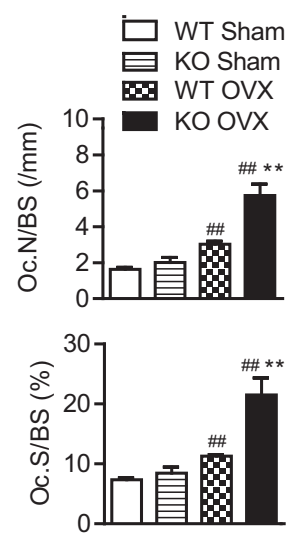

Fig. 3 Aggravation of OVX-induced cancellous bone loss by Sirt6 deficiency. Three-month-old mice were subjected to sham or OVX surgery. a Six weeks after surgery, uterus weight was determined $(n=7-9)$. b The RANKL per OPG ratio in serum was determined $(n=4-5)$. $\mathbf{c}$ Representative $\mu \mathrm{CT}$ reconstruction of cancellous bone in the distal femoral metaphysis. d Representative H\&E staining of the distal femur. Scale bars $=250 \mu \mathrm{m}$. e Representative TRAP staining of the distal femur. Scale bars $=25 \mu \mathrm{m}$. Osteoclast numbers were analyzed in cancellous bone of the distal femur $(n=$ 7 ), and the values are mean \pm SEM. ${ }^{* *} p<0.01$ versus WT; ${ }^{*} p<0.05$ and ${ }^{\# \#} p<0.01$ versus sham. OVX ovariectomy

post-surgery. As expected, in Sirt6 Tg mice, OVX resulted in less bone loss than in the WT mice, whereas no difference in bone mass was noted in mice with sham surgery (Fig. 6a). Consistently, assessment of the cancellous microstructure reflected the inhibitory effects of Sirt6 overexpression on bone resorption (Fig. 6b). These results were further confirmed by ex vivo studies. The FasL-initiated apoptotic pathway was stimulated and osteoclast formation was suppressed in preosteoclasts from Sirt6 $\mathrm{Tg}$ mice compared to those from WT mice (Fig. 6c, d).

\section{Sirt6 deacetylates ERa at Lys171 and Lys299 and increases ERa protein stability}

We investigated the molecular mechanism for ER $\alpha$ regulation by Sirt6, focusing on the relation to its deacetylase activity. HEK293T cells were transfected with plasmid DNA expressing Era and either control vector or Sirt6 and then treated with estrogen (liganded receptor degradation pathway), ICI-182,780 (ER-specific antagonist-induced degradation), or cycloheximide/geldanamycin (CHX/GA, unliganded receptor degradation pathway) [21]. The protein level of ER $\alpha$ gradually decreased in the control group
Twelve-week-old female Sirt6 Tg mice and their littermates were subjected to OVX and sacrificed at 6 weeks 

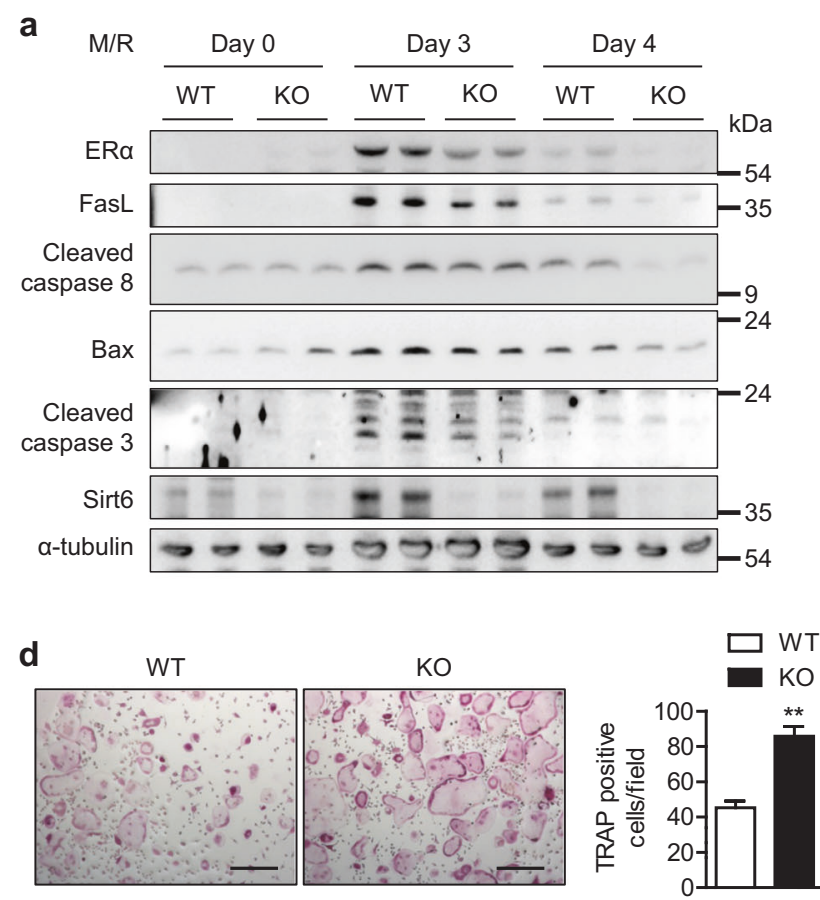

Fig. 4 Increased resistance to apoptotic cell death in preosteoclasts by Sirt6 deficiency. CD11b ${ }^{+}$BMCs from WT and $\mathrm{mS6KO}$ mice were treated with M-CSF $(10 \mathrm{ng} / \mathrm{ml})$ and RANKL $(30 \mathrm{ng} / \mathrm{ml})$ for the indicated time. a Proteins of the extrinsic apoptotic pathway were examined using western blotting. b Two days after osteoclastogenesis induction, the mRNA level of Fasl was determined using real-time RT-PCR $(n=4-5)$. c Three days after osteoclastogenesis induction,

but had a slower rate of decrease in cells overexpressing Sirt6 under each condition tested (Fig. 7a and Fig. S7), indicating that Sirt6 increases ER $\alpha$ stability in both the ligand-dependent and -independent degradation pathways. We hypothesized that ER $\alpha$ upregulation by Sirt6 was due to protein stabilization via proteasome inhibition and repeated the experiments in the presence of the proteasome inhibitor MG132. Indeed, the increased ER $\alpha$ protein level due to Sirt6 overexpression was abolished with MG132 (Fig. S8a). In support of these observations, overexpression of Sirt6 decreased ubiquitination of ER $\alpha$ (Fig. 7b).

Further mechanistic studies using co-immunoprecipitation assays demonstrated a direct interaction between ER $\alpha$ and Sirt6 (Fig. 7c). Moreover, when ER $\alpha$ immunoprecipitates obtained from $\mathrm{CD} 11 \mathrm{~b}^{+}$BMCs lysates were immunoblotted with anti-acetyl lysine (Ac-Lys) antibody, the level of ER $\alpha$ acetylation was increased in mS6KO mice (Fig. 7d). Expression of the deacetylase inactive mutant of Sirt6 had no effects on deacetylation, ubiquitination, or proteasomal degradation of ER $\alpha$ (Fig. S8 and Fig. 7b), indicating that deacetylase activity is required for Sirt6-dependent ER $\alpha$ stabilization. Additional results from luciferase reporter assays using FasL-Luc and ERE-Luc confirmed that ER $\alpha$

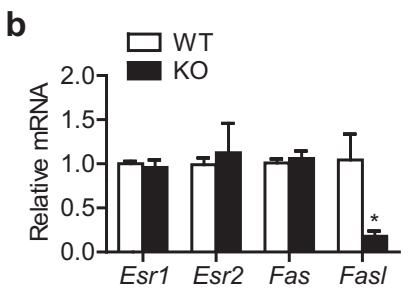

C
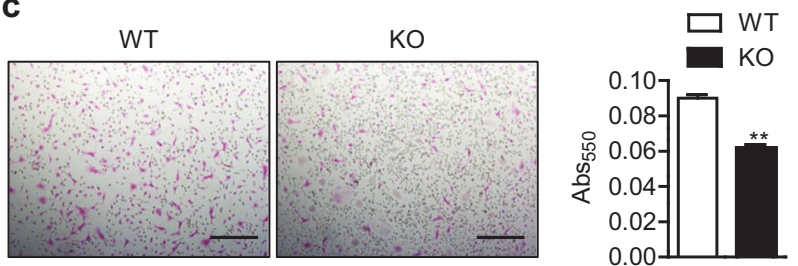

apoptotic cell death was assessed using the APOPercentage apoptosis assay kit. Scale bars $=200 \mu \mathrm{m}$. The absorbance was measured with a spectrophotometer at $550 \mathrm{~nm}(n=10)$. d Four days after osteoclastogenesis induction, cells were stained with TRAP solution. Multinucleate cells with three or more nuclei were scored as osteoclasts per field $(n=5)$. Scale bars $=200 \mu \mathrm{m}$. The values are mean \pm SEM. ${ }^{*} p<$ 0.05 and $* * p<0.01$ versus $\mathrm{WT}$

transcriptional activity was significantly increased by overexpression of Sirt6 but not by that of mSirt6 (Fig. 7e). However, the transcriptional activity of AP-1, which is also located in the Fasl promoter region [22], was not affected by Sirt6.

Next, we sought to identify the Sirt6-dependent deacetylation sites of ER $\alpha$. HEK293T cells were transfected with p300 acetyltransferase with or without Sirt6, and ER $\alpha$ immunoprecipitates from these cells were analyzed using LC-MS/MS. Compared to cells transfected with p300 only, the lysine residues K171 and K299 were deacetylated in cells overexpressing Sir6 (Fig. 8a and Table S2). To determine whether ER $\alpha$ deacetylation by Sirt6 is essential for its protein stability, we substituted each lysine with arginine (K171R and K299R) as a mimic of deacetylated lysine. HEK293T cells were transfected with either WT or mutated $\mathrm{ER} \alpha$ and then treated with estrogen. Under these conditions, the protein stability of KR mutant ER $\alpha$ was significantly increased compared to that of WT ER $\alpha$ (Fig. 8b), indicating that Sirt6 deacetylation of K171 and K299 mediates ER $\alpha$ protein stabilization. Furthermore, FasL promoter activity was significantly elevated in cells containing mutant ER $\alpha$ s, suggesting that deacetylation of 


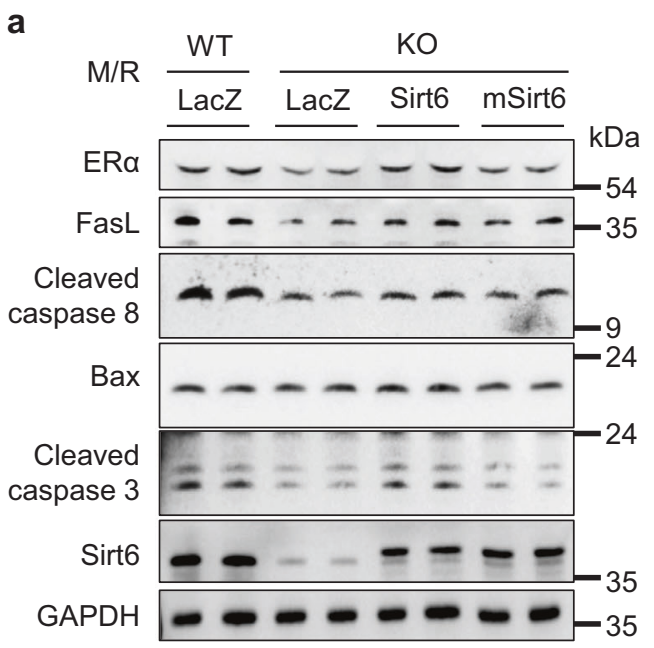

b
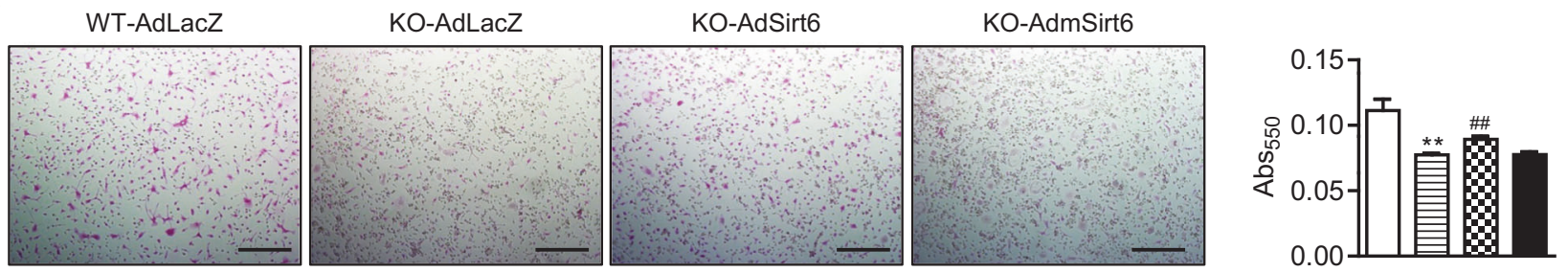

d
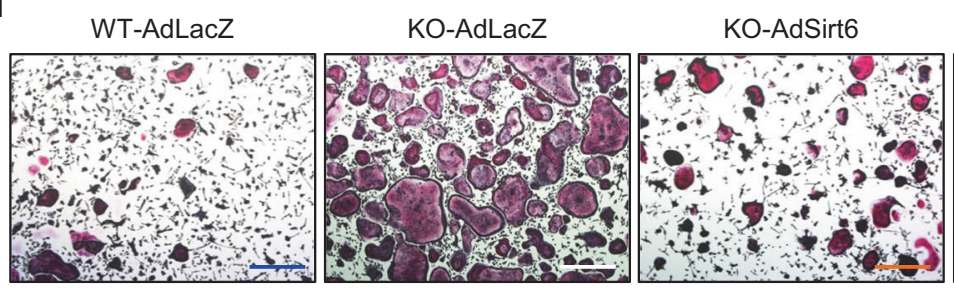

KO-AdmSirt6

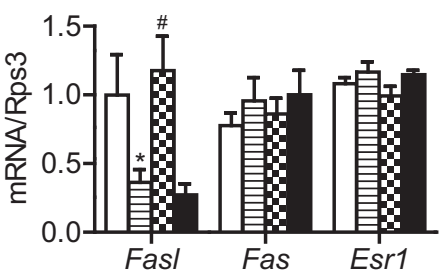

KO-AdLacZ

KO-AdSirt6

KO-AdmSirt6
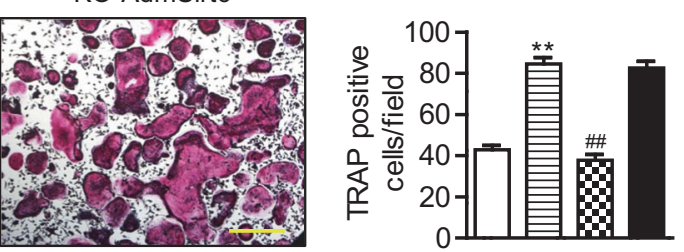

Fig. 5 Restoration of the Sirt6-ER $\alpha$-FasL axis in preosteoclasts by Sirt6 re-expression. CD11b ${ }^{+}$BMCs were transduced with AdLacZ, AdSirt6, or AdmSirt6. a Three days after osteoclastogenesis induction, proteins of the extrinsic apoptotic pathway were examined using western blotting. b Two days after osteoclastogenesis induction, the
mRNA level of Fasl was determined using real-time RT-PCR ( $n=5-$ 6). c, d Apoptotic cell death $(n=4-5)$ and mature osteoclast formation $(n=5)$ were determined as described in the Fig. 4 legend. Scale bars $=200 \mu \mathrm{m}$. The values are mean \pm SEM. $* p<0.05$ and $* * p<0.01$ versus WT-AdLacZ; ${ }^{\#}<0.05$ and ${ }^{\# \#} p<0.01$ versus KO-AdLacZ these lysine residues is critical for the transcriptional activity of ER $\alpha$ (Fig. 8c).

\section{The Sirt6-ERa-FasL axis is suppressed in osteoporotic patients}

To determine the clinical relevance of the above findings, CD11 $\mathrm{b}^{+}$BMCs from human subjects were incubated with M-CSF and RANKL for 7 days, and their Sirt6 and ER $\alpha$ expression was analyzed. Consistent with the findings in mice, the expression of Sirt6 and ER $\alpha$ in human preosteoclasts decreased with age (Fig. 9a, b). Moreover, there were positive correlations between expression of Sirt6 and ER $\alpha$ (Fig. 9c), Sirt6 and bone mineral density (BMD) (Fig. 9d), and ER $\alpha$ and BMD (Fig. 9e), supporting the animal data that Sirt6 increases both ER $\alpha$ protein level and bone mineral density. On the other hand, both Sirt6 and $\mathrm{ER} \alpha$ were negatively correlated with serum C-terminal telopeptide of type 1 collagen (CTX), a marker of osteoclast activity (Fig. 9f, g). As reported, serum CTX was increased in osteoporotic and aged subjects (Figs. S9, S10, and S11). Last, we measured the expression of Sirt6 and $\mathrm{ER} \alpha$ in preosteoclasts from patients with severe osteoporosis and from control subjects. Osteoporotic patients with lower BMD, higher CTX, and older age exhibited significantly lower expression of Sirt6, ER $\alpha$, and FasL in their preosteoclasts compared to control subjects (Fig. 9h, i). These findings suggest that reduced expression of Sirt6 and the resulting decrease of ER $\alpha$ in preosteoclasts contribute to bone loss in osteoporotic patients. 
a

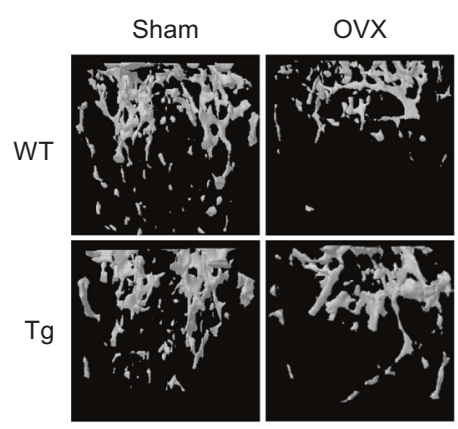

b

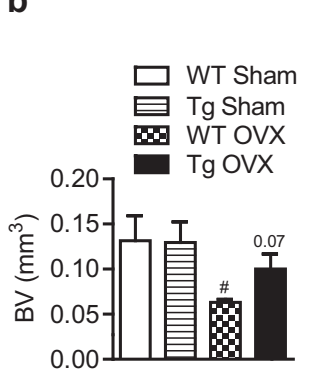

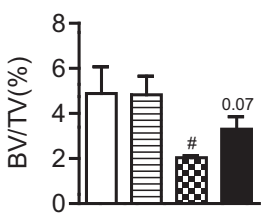
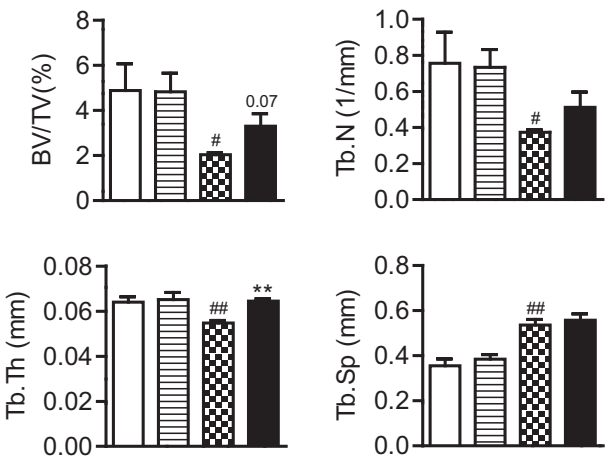

c

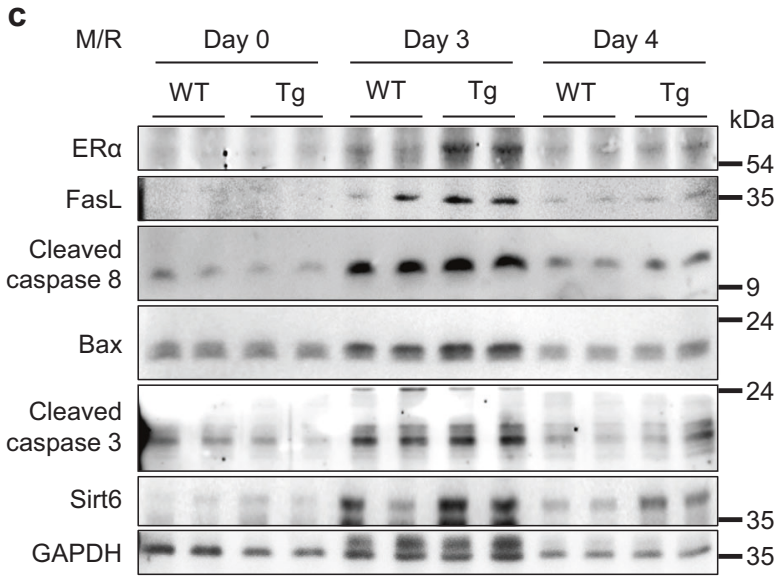

d

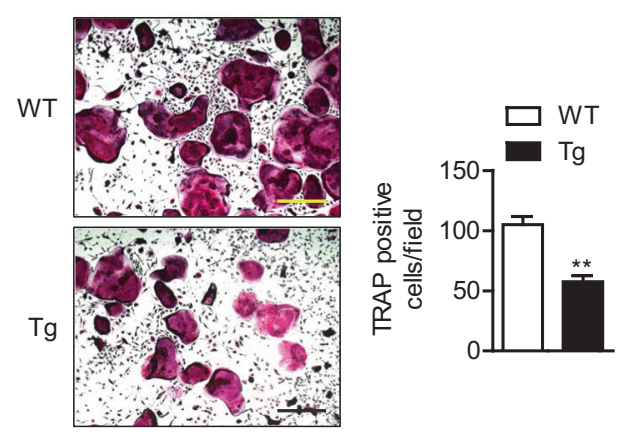

Fig. 6 Preservation of OVX-induced bone loss in Sirt6 Tg mice. a Representative $\mu \mathrm{CT}$ reconstruction of cancellous bone in the distal femur. b Bone morphometric parameters were analyzed $(n=4-6)$. c $\mathrm{CD}_{11 \mathrm{~b}^{+}}$BMCs from WT or Sirt6 Tg mice were treated with M-CSF $(10 \mathrm{ng} / \mathrm{ml})$ and RANKL $(30 \mathrm{ng} / \mathrm{ml})$ for the indicated times, and

\section{Discussion}

Using two different but complementary animal models, we demonstrated that Sirt6 reduces OVX- and aging-related bone loss by deacetylating $\mathrm{ER} \alpha$, revealing a previously uncharacterized posttranslational regulation of ER $\alpha$ stability by Sirt6. Specifically, deletion of Sirt6 in myeloid cells enhanced osteoclastogenesis and reduced cancellous bone mass after OVX, which was similar to what is seen in myeloid ER $\alpha$ KO mice [6]. Similar observations were made in aged mS6KO mice. Concordantly, these phenotypes were completely abrogated in Sirt6 Tg mice. Analysis of human BMC samples confirmed lower expression of Sirt6 and ER $\alpha$ in preosteoclasts from osteoporotic patients compared with those from control subjects, suggesting that Sirt6 is a negative regulator of osteoporosis in human.

Osteoporosis is a multifactorial disease mainly caused by a combination of aging and hormone deficiency [20, 23]. Although OVX is the preferred animal model to study osteoporosis, OVX performed in young animals does not completely recapitulate osteoporotic phenotypes observed proteins of the extrinsic apoptotic pathway were examined using western blotting. d Representative TRAP staining after osteoclastogenesis induction. Scale bars $=200 \mu \mathrm{m}$. The TRAP-positive cells were counted per field $(n=6)$, and the values are mean \pm SEM. ${ }^{\#} p<0.05$ and ${ }^{\# \#} p<0.01$ versus WT Sham. ${ }^{*} p<0.01$ versus WT or WT OVX

in old subjects, suggesting the presence of distinct regulatory mechanism between estrogen deficiency- and aginginduced bone loss. Nevertheless, based on the following evidence, we suggest that Sirt6 is a common protective factor against aging- and estrogen deficiency-induced osteoporosis. First, Sirt6 deletion in preosteoclasts augmented bone loss in both aging- and ovariectomy-induced osteoporosis models. Second, the bone morphometric phenotypes of ovariectomized $\mathrm{mS6KO}$ mice were similar to those of old $\mathrm{mS6KO}$ mice. In both models, bone loss was limited to cancellous bone. Third, the impairment of the Sirt6-ER $\alpha$-FasL axis seen in preosteoclasts from mS6KO mice was also observed in those from old mice.

One regulatory mechanism of osteoclastogenesis is apoptosis, and the bone-sparing action of estrogen is related to its activity on osteoclastic apoptosis $[4,5]$. In mice, FasL expression in osteoclasts is likely to be the mechanism by which Sirt6 promotes osteoclast apoptosis through the following pathway: Sirt6 deficiency in preosteoclasts $\rightarrow$ decreased ER $\alpha$ expression $\rightarrow$ decreased FasL expression $\rightarrow$ decreased activity of the extrinsic 

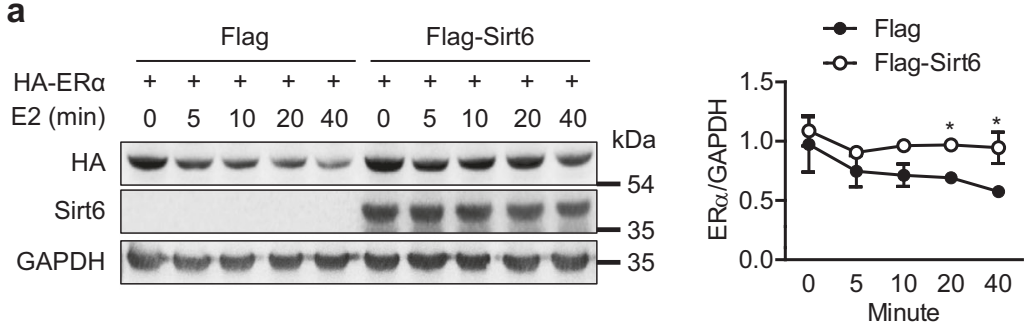

C

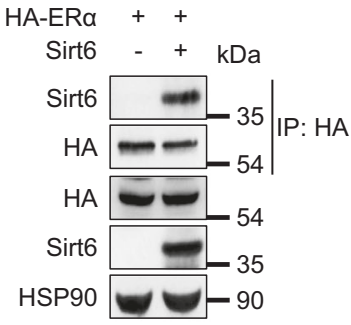

d

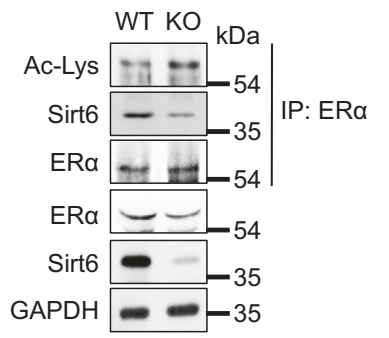

b
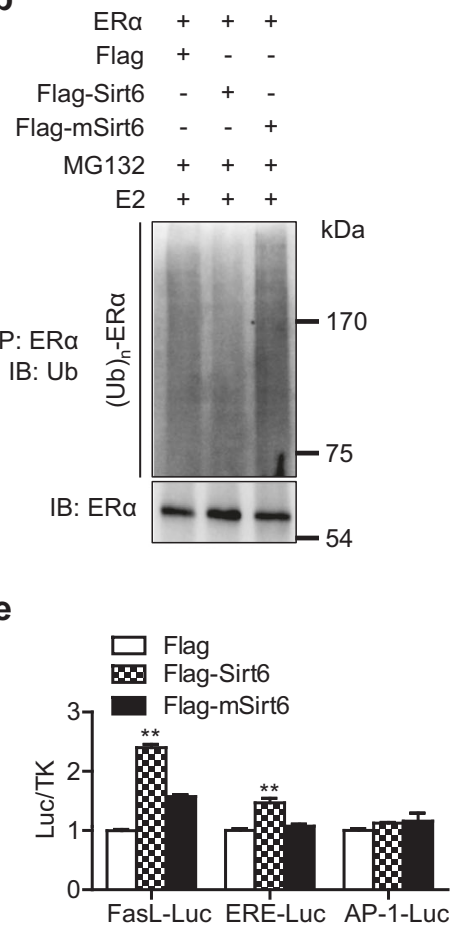

Fig. 7 Blockade of ubiquitin-proteasomal degradation of ER $\alpha$ by Sirt6. a HEK293T cells transfected with Era were treated with estrogen (E2, $10 \mathrm{nM}$ ) for the indicated time, and the relative protein levels of ER $\alpha$ were compared $(n=3)$. b HEK293T cells were transfected with Era and Sirt6 or deacetylase-lacking mutant Sirt6 (mSirt6) as indicated. After $24 \mathrm{~h}$, the cells were treated with E2 $(10 \mathrm{nM})$ for $40 \mathrm{~min}$ in the presence of MG132 $(2 \mu \mathrm{M})$, and total cell lysates were immunoprecipitated with anti-ER $\alpha$ antibodies and immunoblotted with antiubiquitin antibodies. c After transfection in HEK293T cells as indicated, a co-immunoprecipitation assay to determine the physical interaction between Sirt6 and ER $\alpha$ was performed. d CD11b ${ }^{+}$BMCs from WT or mS6KO mice were treated with M-CSF and RANKL for 3 days, and whole cell lysates were immunoprecipitated with anti-ER $\alpha$ antibody and subjected to western blot to detect ER $\alpha$ acetylation. e HEK293T cells were transfected with WT or mutant Sirt6, and the FasL, ERE and AP1 luciferase activity in the cell lysates was measured $(n=4)$. The values are mean \pm SEM. $* p<0.05$ and $* * p<$ 0.01 versus Flag apoptotic pathway $\rightarrow$ increased number of $\mathrm{TRAP}^{+}$ osteoclasts. Overall, these effects favor bone resorption. The increased resistance to apoptosis in osteoclasts that occurs with advancing age is also related to downregulation of FasL expression. This view is further supported by evidence that transgenic Sirt6 overexpression has the opposite effects (i.e., promoting the FasL-initiated apoptosis of osteoclasts and increasing cancellous bone mass). These results are congruent with those of Nakamura et al. [4], who showed that osteoclasts are the source of FasL in the stimulatory effect of estrogen on osteoclast apoptosis.

Considering that sirtuins are tightly linked with aging and the associated pathology, we investigated changes in expression of sirtuin-family proteins in preosteoclasts. Upon osteoclastogenesis induction, only Sirt6 expression was significantly decreased in preosteoclasts from old mice compared to those from young mice. Consistent with the animal data, Sirt6 expression in human preosteoclasts was also decreased with advancing age, suggesting that old subjects who express low levels of Sirt6 in preosteoclasts would be more susceptible to osteoclastic bone resorption than young subjects.

As described, $\mathrm{ER} \alpha$ is a well-known target for posttranslational modifications, and four potential sites for acetylation have been reported in the hinge region of ER $\alpha$ (K266, K288, K302, and K303) [24]. This acetylation is a reversible process, and balance between acetylation and deacetylation is a regulatory mechanism for determining $\mathrm{ER} \alpha$ activity [11, 13]. To our knowledge, the regulatory mechanism for $\mathrm{ER} \alpha$ deacetylation and its biological significance have not yet been reported. We identified Sirt6 as a deacetylase of $\mathrm{ER} \alpha$, with deacetylation occurring at K171 in the AF1 domain and K299 in the hinge region. Multiple lines of evidence support a direct link of Sirt6-mediated deacetylation of $\mathrm{ER} \alpha$ to its protein stability. First, deacetylation of $\mathrm{ER} \alpha$ by Sirt6 decreased ubiquitination and subsequent proteasomal degradation, which is consistent with the effect of MG132 treatment [25]. Second, Sirt6 transfection increased the transcriptional activity of $\mathrm{ER} \alpha$, whereas Sirt6 deletion yielded the opposite results. Finally, lysine-to-arginine substitutions (K171R and 

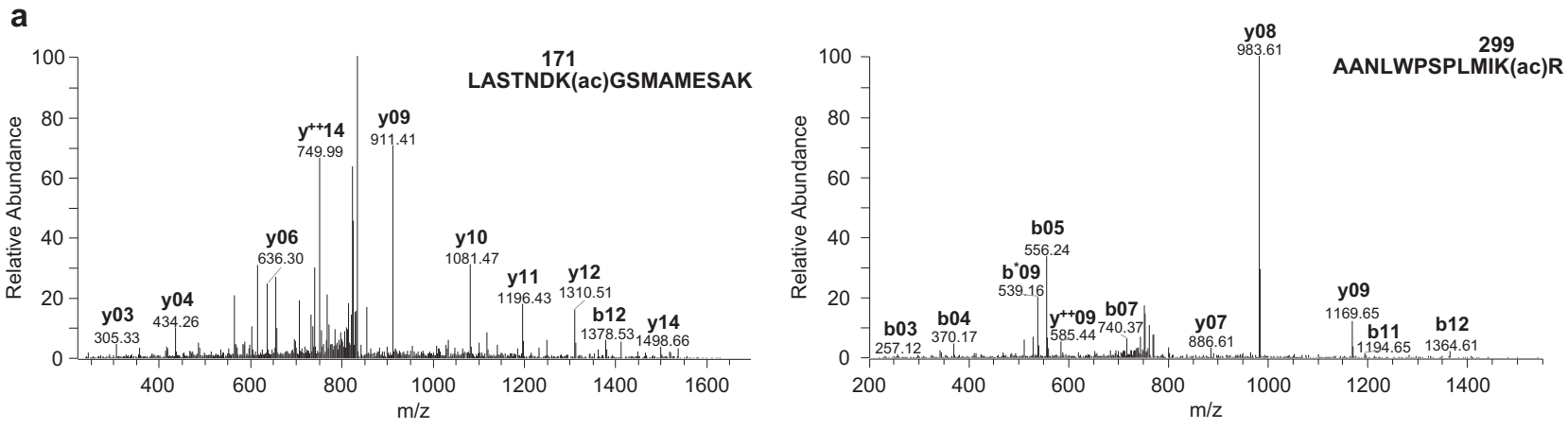

b

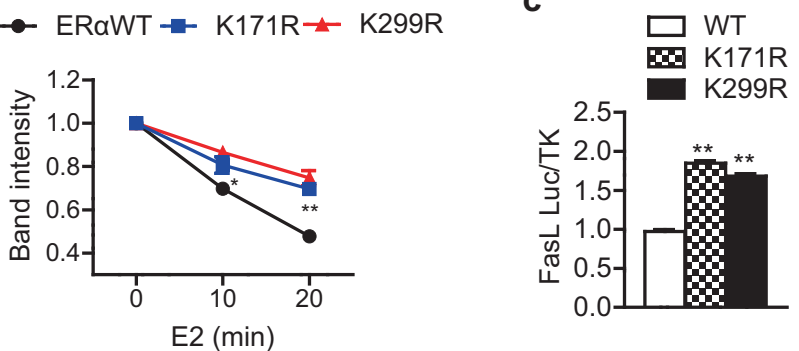

Fig. 8 Identification of the Sirt6-mediated deacetylation sites of ER $\alpha$. a HEK293T cells were transfected with Era and p300 with or without Sirt6. Acetylation of ER $\alpha$ was determined using LC-MS/MS analysis after in-gel digestion. MS/MS spectra of acetylated ER $\alpha$ peptides containing Lys171 (left) and Lys299 (right) are shown. b HEK293T cells transfected with wild (WT) or mutant Era (K171R or

K299R) at the deacetylation sites augmented the protein stability and transcriptional activity of ER $\alpha$ compared to WT ER $\alpha$. Collectively, these evidence demonstrate that deacetylation of $E R \alpha$ by Sirt6 is a novel regulatory mechanism of ER $\alpha$-triggered anti-osteoporotic signaling. Although we hypothesize that Sirt6 directly affects the stability and activity of ER $\alpha$ in preosteoclasts, we cannot exclude the possibility that Sirt6 may influence ER $\alpha$ target gene expression by modulating chromatin structure by histone deacetylation.

Although this study has some possible limitations, there are a number of strengths. (1) The use of two different models of osteoporosis enabled us to study the role of Sirt6 on estrogen deficiency- and aging-associated bone loss. (2) Using genetic loss- and gain-of-function mutations in the Sirt6, we confirmed the role of Sirt6 in osteoclast precursor cells, both in terms of maturation and apoptosis, in bone remodeling subsequent to OVX. (3) Comparative analysis of human preosteoclasts derived from BMCs isolated from osteoporotic patients and control subjects demonstrated the clinical relevance of the study. (4) Identification of ER $\alpha$ as a new non-histone deacetylation target of Sirt6 is of great importance in sirtuin biology. Because of its nuclear localization, a great part of the biological activity of Sirt6 has previously been explained by deacetylation of histone on H3K9, H3K56, and H3K18. (5) The elucidation of ER $\alpha$

K299R) were treated with $\mathrm{E} 2(10 \mathrm{nM})$ for the indicated time. The relative protein levels of $\mathrm{ER} \alpha$ were compared $(n=4)$. c HEK293T cells were transfected with WT or mutant Era, and FasL luciferase activity was determined $(n=4)$. The values are mean \pm SEM. $* p<0.05$ and $* * p<0.01$ versus WT

function in the suppression of bone resorption irrespective of estrogen suggests a new therapeutic target to replace hormone therapy, which has side effects to reproductive organs. However, we did not compare the bone mass parameters between aged Sirt6 Tg and WT mice due to the unavailability of 20-month-old Sirt6 Tg mice. A future study to confirm the beneficial effects of Sirt6 overexpression and/or activation on preservation of bone mass in aged mice is needed.

In summary, we propose a model to explain the molecular mechanism of Sirt6-mediated ER $\alpha$ deacetylation and osteoporosis prevention (Fig. S12). In young subjects with estrogen deficiency, Sirt6 deacetylates ER $\alpha$ and maintains its protein stability. Consequently, expression of the ER $\alpha$ target gene FasL is increased, which causes apoptotic cell death of preosteoclasts possibly in an autocrine or paracrine manner. However, in subjects with advanced age and estrogen deficiency, Sirt6 protein level and the ER $\alpha$-FasL axis are downregulated, which leads to enhanced formation of mature osteoclasts. The acetylation status of ER $\alpha$ seems to serve as a convergence point for cellular responses to aging, with or without estrogen deficiency, and for development of osteoporosis. Therefore, targeting ER $\alpha$ in senescent osteoclasts with a Sirt6 agonist would be a novel strategy for preventing and treating osteoporosis by reducing bone resorption. 

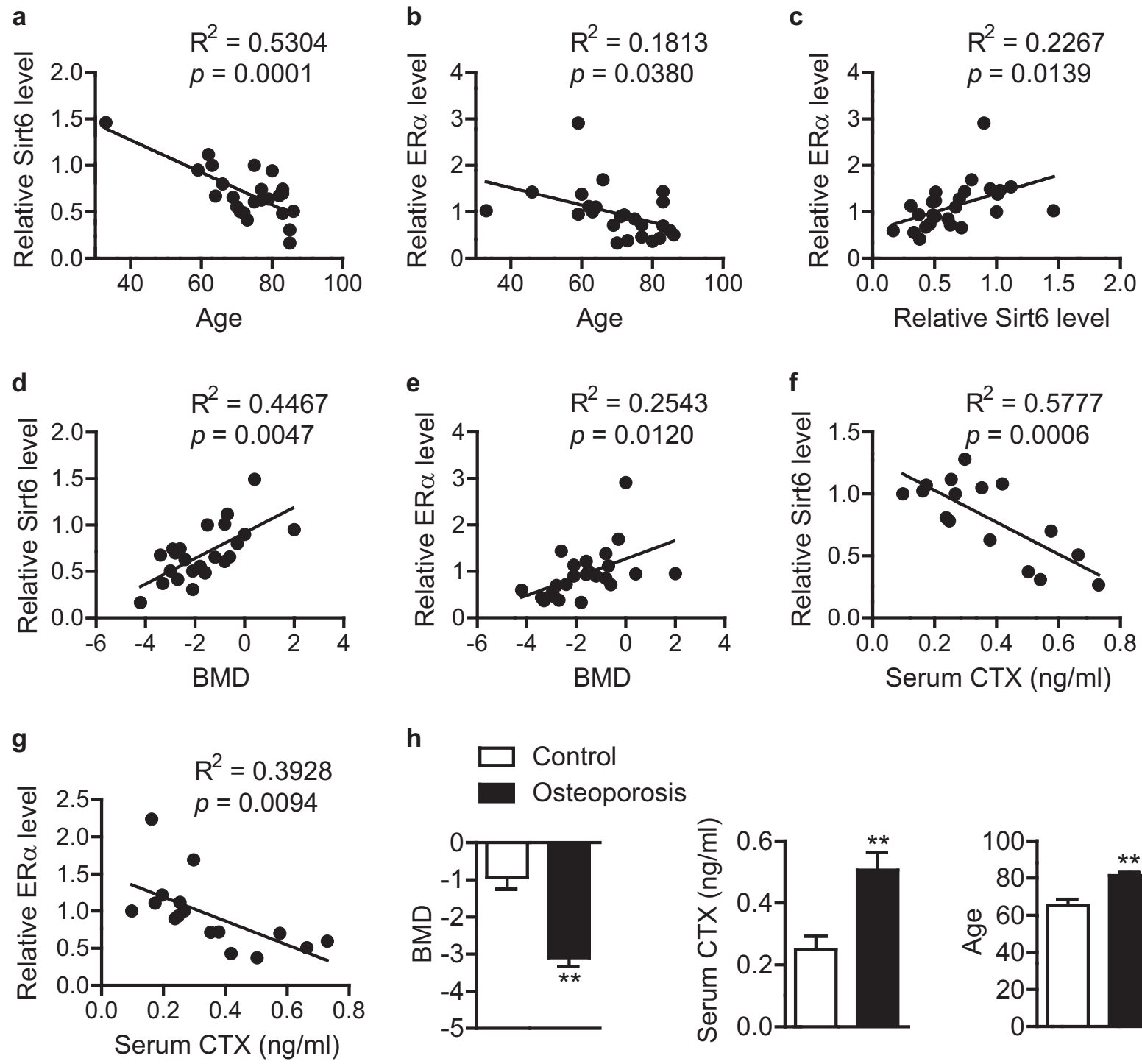

h
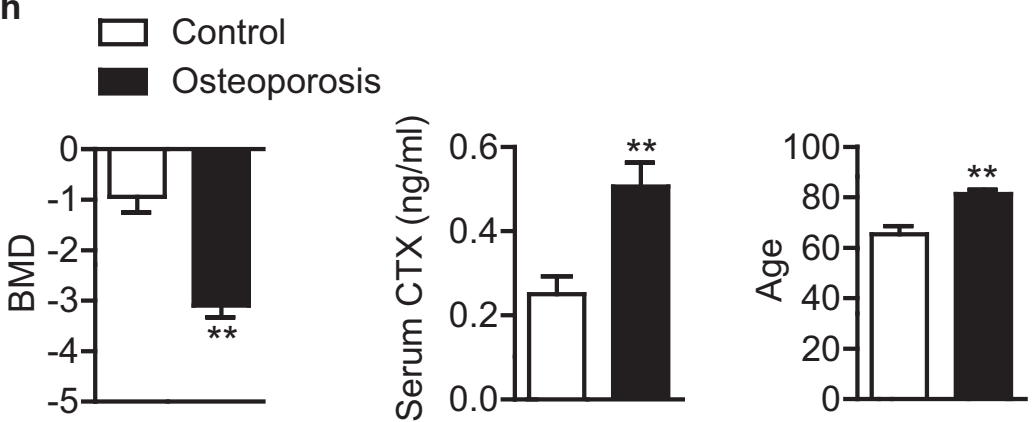

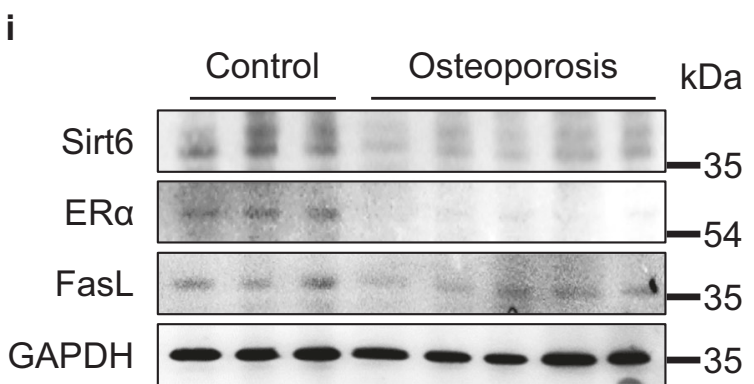

Fig. 9 Attenuation of the Sirt6-ER $\alpha$-FasL axis in severe osteoporosis patients. Human CD11b ${ }^{+}$BMCs were treated with M-CSF $(25 \mathrm{ng} / \mathrm{ml})$ and RANKL $(50 \mathrm{ng} / \mathrm{ml})$ for 7 days. a-g Scatter plots of Sirt6 expression and various bone parameters. The coefficient of determination was used to compare the association of Sirt6 expression with bone parameters

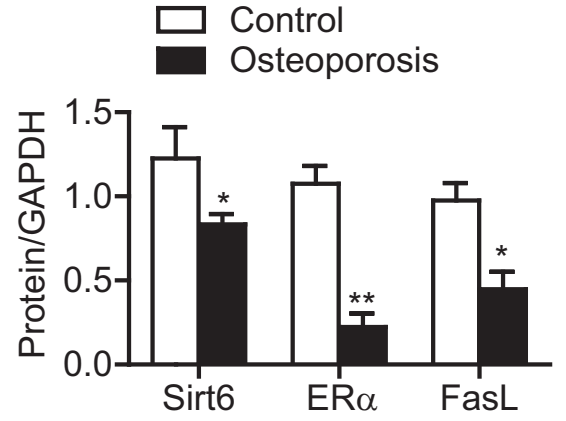

$(\mathbf{a}-\mathbf{e}, n=24 ; \mathbf{f}-\mathbf{g}, n=16) . \mathbf{h}$ Clinical parameters of control subject and osteoporotic patients. i Human CD11b ${ }^{+}$BMCs were treated with M-CSF and RANKL for 7 days, and the expression of Sirt6, ER $\alpha$, and FasL proteins was analyzed using western blotting. The values of $\mathbf{h}$ and $\mathbf{i}$ are mean \pm SEM. $* p<0.05$ and $* * p<0.01$ versus control 
Acknowledgements This work was supported by grants from the Medical Research Center Program (NRF-2017R1A5A2015061) through the National Research Foundation (NRF), which is funded by the Korean government (MSIP).

Author contributions YJM, ZZ, IHB, OKK, and SJY performed the experiments and analyzed the data. JRK and SL interpreted the data. EJB and BHP designed the experiments, interpreted the data, and wrote the manuscript. All authors reviewed the manuscript.

\section{Compliance with ethical standards}

Conflict of interest The authors declare that they have no conflict of interest.

Publisher's note: Springer Nature remains neutral with regard to jurisdictional claims in published maps and institutional affiliations.

\section{References}

1. Manolagas SC. From estrogen-centric to aging and oxidative stress: a revised perspective of the pathogenesis of osteoporosis. Endocr Rev. 2010;31:266-300.

2. Looker AC, Wahner HW, Dunn WL, Calvo MS, Harris TB, Heyse SP, et al. Updated data on proximal femur bone mineral levels of US adults. Osteoporos Int. 1998;8:468-89.

3. Almeida M, Han L, Martin-Millan M, Plotkin LI, Stewart SA, Roberson PK, et al. Skeletal involution by age-associated oxidative stress and its acceleration by loss of sex steroids. J Biol Chem. 2007;282:27285-97.

4. Nakamura T, Imai Y, Matsumoto T, Sato S, Takeuchi K, Igarashi $\mathrm{K}$, et al. Estrogen prevents bone loss via estrogen receptor alpha and induction of Fas ligand in osteoclasts. Cell. 2007;130:811-23.

5. Krum SA, Miranda-Carboni GA, Hauschka PV, Carroll JS, Lane TF, Freedman LP, et al. Estrogen protects bone by inducing Fas ligand in osteoblasts to regulate osteoclast survival. EMBO J. 2008;27:535-45.

6. Martin-Millan M, Almeida M, Ambrogini E, Han L, Zhao H, Weinstein RS, et al. The estrogen receptor-alpha in osteoclasts mediates the protective effects of estrogens on cancellous but not cortical bone. Mol Endocrinol. 2010;24:323-34.

7. Tecalco-Cruz AC, Ramirez-Jarquin JO. Mechanisms that increase stability of estrogen receptor alpha in breast cancer. Clin Breast Cancer. 2017;17:1-10.

8. Cui Y, Zhang M, Pestell R, Curran EM, Welshons WV, Fuqua SA. Phosphorylation of estrogen receptor alpha blocks its acetylation and regulates estrogen sensitivity. Cancer Res. 2004;64:9199-208.

9. Sentis S, Le Romancer M, Bianchin C, Rostan MC, Corbo L. Sumoylation of the estrogen receptor alpha hinge region regulates its transcriptional activity. Mol Endocrinol. 2005;19:2671-84.

10. La Rosa P, Pesiri V, Leclercq G, Marino M, Acconcia F. Palmitoylation regulates $17 \beta$-estradiol-induced estrogen receptor- $\alpha$ degradation and transcriptional activity. Mol Endocrinol. 2012;26:762-74.

11. Kim MY, Woo EM, Chong YT, Homenko DR, Kraus WL. Acetylation of estrogen receptor alpha by p300 at lysines 266 and
268 enhances the deoxyribonucleic acid binding and transactivation activities of the receptor. Mol Endocrinol. 2006;20:1479-93.

12. Wang $\mathrm{C}, \mathrm{Fu} \mathrm{M}$, Angeletti RH, Siconolfi-Baez L, Reutens AT, Albanese C, et al. Direct acetylation of the estrogen receptor alpha hinge region by $\mathrm{p} 300$ regulates transactivation and hormone sensitivity. J Biol Chem. 2001;276:18375-83.

13. Kim SH, Kang HJ, Na H, Lee MO. Trichostatin A enhances acetylation as well as protein stability of ERalpha through induction of p300 protein. Breast Cancer Res. 2010;12:R22.

14. Imai S, Guarente L. NAD+ and sirtuins in aging and disease. Trends Cell Biol. 2014;24:464-71.

15. Zainabadi K, Liu CJ, Caldwell ALM, Guarente L. SIRT1 is a positive regulator of in vivo bone mass and a therapeutic target for osteoporosis. PLoS ONE. 2017;12:e0185236.

16. Hah YS, Cheon YH, Lim HS, Cho HY, Park BH, Ka SO, et al. Myeloid deletion of SIRT1 aggravates serum transfer arthritis in mice via nuclear factor- $\kappa B$ activation. PLoS ONE. 2014;9:e87733.

17. Artsi H, Cohen-Kfir E, Gurt I, Shahar R, Bajayo A, Kalish N, et al. The Sirtuin1 activator SRT3025 down-regulates sclerostin and rescues ovariectomy-induced bone loss and biomechanical deterioration in female mice. Endocrinology. 2014; 155:3508-15.

18. Pearson KJ, Baur JA, Lewis KN, Peshkin L, Price NL, Labinskyy $\mathrm{N}$, et al. Resveratrol delays age-related deterioration and mimics transcriptional aspects of dietary restriction without extending life span. Cell Metab. 2008;8:157-68.

19. Kawahara TL, Michishita E, Adler AS, Damian M, Berber E, Lin M, et al. SIRT6 links histone H3 lysine 9 deacetylation to $\mathrm{NF}-\kappa \mathrm{B}-$ dependent gene expression and organismal life span. Cell. 2009;136:62-74.

20. Manolagas SC. The quest for osteoporosis mechanisms and rational therapies: how far we've come, how much further we need to go. J Bone Miner Res. 2018;33:371-85.

21. Berry NB, Fan M, Nephew KP. Estrogen receptor-alpha hingeregion lysines 302 and 303 regulate receptor degradation by the proteasome. Mol Endocrinol. 2008;22:1535-51.

22. Mor G, Sapi E, Abrahams VM, Rutherford T, Song J, Hao XY, et al. Interaction of the estrogen receptors with the Fas ligand promoter in human monocytes. J Immunol. 2003;170:114-22.

23. Ucer S, Iyer S, Kim HN, Han L, Rutlen C, Allison K, et al. The effects of aging and sex steroid deficiency on the murine skeleton are independent and mechanistically distinct. J Bone Miner Res. 2017;32:560-74.

24. Manolagas SC, O'Brien CA, Almeida M. The role of estrogen and androgen receptors in bone health and disease. Nat Rev Endocrinol. 2013;9:699-712.

25. Reid G, Hubner MR, Metivier R, Brand H, Denger S, Manu D, et al. Cyclic, proteasome-mediated turnover of unliganded and liganded ERalpha on responsive promoters is an integral feature of estrogen signaling. Mol Cell. 2003;11:695-707.

26. Lee Y, Ka SO, Cha HN, Chae YN, Kim MK, Park SY, et al. Myeloid sirtuin 6 deficiency causes insulin resistance in high-fat diet-fed mice by eliciting macrophage polarization toward an M1 phenotype. Diabetes. 2017;66:2659-68.

27. Qiang L, Wang L, Kon N, Zhao W, Lee S, Zhang Y, et al. Brown remodeling of white adipose tissue by SirT1-dependent deacetylation of Ppary. Cell. 2012;150:620-32. 